\title{
Editorial
}

\section{ALIANZA INTERNACIONAL PARA LA GLOBALIZACIÓN: EL POLITÉCNICO GRANCOLOMBIANO INGRESA A WHITNEY INTERNATIONAL UNIVERSITY SYSTEM}

La Fundación Politécnico Grancolombiano, Institución Universitaria, acaba de iniciar una de las etapas más importantes de su historia al vincularse como miembro de la red de instituciones de educación superior Whitney International University System.

En lo económico y social, según el Informe de Desarrollo Humano del Programa de las Naciones Unidas para el Desarrollo Humano (PNUD), "el ingreso total de las 500 personas más ricas del mundo es superior al ingreso de los 416 millones de personas más pobres. Los 2.500 millones que viven con menos de dos dólares al día -que representan el 40 por ciento de la población mundial- obtienen sólo el 5 por ciento del ingreso mundial. El 10 más rico, consigue el 54 por ciento. Mil millones de personas sobreviven en la extrema pobreza con menos de un dólar diario, más de 10 millones de niños no alcanzan a cumplir 5 años a causa de muertes que se podrían haber evitado, todavía hay 115 millones de menores que no asisten a la escuela, más de 1.000 millones de personas no tienen agua potable, 2.600 millones no tienen acceso al saneamiento, y el VIH sigue cobrando las vidas de millones de personas. El Informe revela que hoy día una persona que vive en Zambia tiene menos probabilidades de llegar a los 30 años que una persona que vivía en Inglaterra en 1840. Y esos son sólo unos pocos datos".(Resumen tomado de la Revista Semana, No. 1.219, página 100).

En lo ecológico estamos destruyendo el medio ambiente lenta, pero seguramente. Las desigualdades sociales generan toda suerte de conflictos violentos. Las armas nucleares permitirían a unas pocas naciones, incluyendo algunas subdesarrolladas, destruir la humanidad en pocos segundos. Las ventajas del increíble desarrollo científico, tecnológico y cultural alcanzado por la sociedad humana benefician realmente a un porcentaje minoritario de la población mundial. En lo político, los conflictos sociales y los intereses económicos de las clases sociales predominantes amenazan gravemente los sistemas democráticos. La capacidad del ordenamiento jurídico internacional para resolver los conflictos entre países o para proteger los derechos humanos es muy limitada. En lo cultural, existe una gran supremacía de los bienes culturales con mercado, es decir, la cultura basada en el rating, sobre los valores de la bondad y el amor al prójimo, que, para que no se les dé solamente una connotación religiosa, prefiero denominar sentido de solidaridad, equidad y generosidad. El dinero es el valor fundamental de la actual civilización.

En suma, aunque el ser humano está compelido a no perder la esperanza, si se hiciera un estudio de factibilidad descarnado, resultaría 
que la humanidad no es viable, empezando porque existe un descomunal déficit del principal insumo de la realización humana, que es la educación.

A mi juicio los sistemas educativos no están respondiendo adecuadamente a las necesidades del desarrollo social y económico del mundo. Representémonos el sistema educativo global o de cualquier país como una pirámide dividida en varias franjas, y tomemos como ejemplo el caso Colombiano. La pirámide colombiana se vería así:

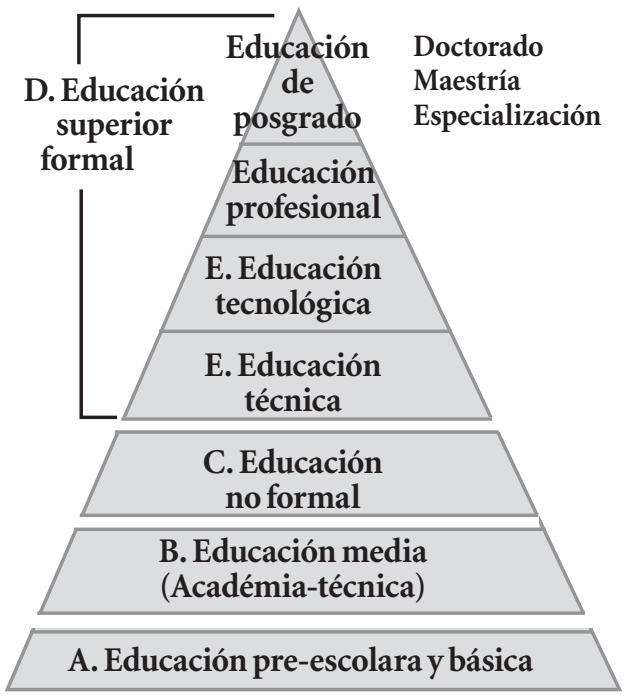

En el año 2005 el país todavía registraba un índice de analfabetismo relativamente alto, alrededor de un 6,7\%. En la educación preescolar la cobertura es inferior al $50 \%$ y la calidad pedagógica es todavía muy deficiente. En educación básica primaria y secundaria, es decir, hasta el grado $9^{\circ}$, aunque los niveles de cobertura se acercan al $100 \%$, las pruebas SABER del propio Gobierno Nacional arrojan resultados muy preocupantes. No más del $20 \%$ de los estudiantes de grado $9^{\circ}$ pueden hacer la lectura comprensiva de un texto; no más del 11\% manejan adecuadamente las operaciones matemáticas básicas, y, en general, tienen una formación muy deficiente en competencias ciudadanas. Para el año 2004, en los resultados del examen de estado del ICFES el 70\% de los colegios se encontraban en las categorías media, baja, inferior y muy inferior, en tanto que sólo el 30\% restante se ubica en los niveles alto, superior y muy superior.

En educación media, o sea los grados $10^{\circ}$ y $11^{\circ}$, la cobertura es baja, del $77,9 \%$ en el año 2005 , y hay graves problemas de calidad y pertinencia. La mayoría de los estudiantes, cerca del $65 \%$, se orienta hacía el bachillero clásico o académico, pues su gran ambición, dentro de nuestros patrones culturales, es ingresar posteriormente a la universidad para cursar una carrera profesional de cinco (5) años, y que posteriormente le digan “doctor" sin realmente serlo. Es los que llamamos la falsa doctoritis, que es una ridícula costumbre colombiana. El otro 35\% de los estudiantes ingresa a la educación media técnica vocacional, cuyo propósito es prepararlos para la vida laboral en algún oficio específico. En Colombia hay alrededor de 50 modalidades de media técnica vocacional, pero solamente cuatro, la comercial, la industrial, la agropecuaria y la pedagógica, han tenido un desarrollo significativo, y, en general, su éxito en la formación para el trabajo ha sido escaso, pues los egresados no consiguen sino empleos de mala calidad.

En Colombia existen cerca de 11 millones de jóvenes que no encuentran oportunidades adecuadas de trabajo, ni de educación superior. Para ellos es fundamental contar con un sistema sólido de educación no formal que sirva de puente entre la educación secundaria y la vida laboral o, eventualmente, la educa- 
ción superior, el cual en nuestro país es muy débil. En Bogotá, por ejemplo, donde existe la mayor necesidad, hay aproximadamente 1.200 instituciones de educación no formal, pero solamente unas 900 registradas ante las autoridades distritales, y la mayoría son empresas débiles y menospreciadas por la sociedad, que no cuentan con un indispensable sistema de certificación de competencias para el trabajo, el cual les serviría para legitimar su valiosa función.

En cuanto a la educación superior, que conforma la parte más alta de la pirámide, también a su vez dividida en varias franjas, Colombia tiene una cobertura bajísima, de alrededor del 25\%, que apenas se acerca al promedio latinoamericano y un altísimo nivel de deserción, superior al 50\%, en las carreras de cinco (5) años. En el país apenas se empieza a desarrollar un sistema de aseguramiento de la calidad, por medio de los registros calificados de los programas académicos ante el Ministerio de Educación y de la acreditación voluntaria tanto de programas académicos como de instituciones, por parte del Consejo Nacional de Acreditación, CNA, como organismo independiente. A mi juicio, los criterios de evaluación de la calidad académica están mal enfocados, pues les hace falta el concepto de eficiencia. El sistema de educación superior colombiano, al igual que el de casi todo el mundo, está signado por el paradigma de profesores que hablan frente de estudiantes que duermen y después se "clavan" solamente tres días para pasar un examen ¿De qué sirve tener tantos edificios, bibliotecas, computadores y profesores de tiempo completo, si los alumnos no estudian continuamente y no sienten la necesidad de forjar una mente investigativa? Este paradigma implica un gran desperdicio de recursos humanos, y constituye una de las razones más importantes de que la cobertura de la educación superior sea tan baja.

Dentro de la educación superior, los niveles técnico y tecnológico, es decir, la educación integral pero práctica para el trabajo, están especialmente descuidados en Colombia y en muchos otros países. Las instituciones de educación técnica y tecnológica son débiles en lo institucional, lo patrimonial y lo académico, $y$, en general, no ofrecen verdadera formación práctica, sino de carácter universitario, aunque de menor duración, en disciplinas blandas, con poco uso de laboratorios, los cuales resultan demasiado costosos para las instituciones. El SENA (Servicio Nacional de Aprendizaje) realiza grandes esfuerzos, pero no da abasto para las enormes necesidades del país. Las instituciones de educación técnica y tecnológica son menospreciadas por la sociedad y estigmatizadas como universidades de "garaje", y la educación técnica y tecnológica es considerada como de menor categoría, pues conduce a empleos de inferior remuneración, como lo reflejan los primeros resultados del Observatorio Laboral, sin que las remuneraciones de los profesionales de cinco años sean muy satisfactorias.

La investigación es una de las funciones u obligaciones más cacareadas de la educación superior y de las universidades en el mundo actual. La sociedad contemporánea está incurriendo en la ingenuidad de exigirles investigación aplicada a todas las instituciones en todos los niveles, a pesar de que la mayoría no tienen recursos para realizarla. Ni siquiera los programas académicos de posgrado, o sea la cúspide de la pirámide, que debieran tener el mayor componente de investigación, logran impactar a la sociedad o a la economía con nuevos conocimientos o tecnologías, pues en general se limitan a desarrollar currículos 
teóricos de simple complementación académica. Incluso en los países desarrollados, como los Estados Unidos y los europeos, son pocas las universidades que tienen capacidad para realizar investigación de frontera, $y$ por lo general dependen de recursos gubernamentales o de las grandes corporaciones privadas para este propósito. Es por todo esto por lo que las principales funciones de la universidad contemporánea son de carácter formativo $y$, desde el punto de vista de la función investigativa, lo más importante es la investigación formativa, es decir, el carácter investigativo o inquisitivo del proceso pedagógico en cualquier programa académico, lo cual resulta imposible dentro del mencionado paradigma de los estudiantes pasivos que duermen enfrente de los profesores que hablan.

Por estas razones tan fundamentales, relativas a todas las franjas de la pirámide del sistema educativo en Colombia y en el mundo, la sociedad contemporánea está demandando una gran revolución en la universidad y en la educación superior, cuyos objetivos centrales deben ser romper ese patrón del alumno pasivo y buscar la responsabilidad académica y la autonomía intelectual, tanto del estudiante como del profesor. Ninguna institución educativa del mundo que quiera ser exitosa podrá eludir esta revolución.

La Fundación Politécnico Grancolombiano, Institución Universitaria, viene luchando desde hace un tiempo considerable por tales objetivos, pero sin duda la alianza con Whitney International University System le dará un significativo impulso a esa revolución de la responsabilidad académica y la autonomía intelectual de la comunidad académica. Con esta ayuda la Institución afianzará su propósito de graduar estudiantes especialmente hábiles para la identificación y la solución de problemas.

Whitney International University System es una red internacional de universidades, que se propone unir universidades de todo el mundo para perfeccionar sus procesos académicos y administrativos, para aumentar la cobertura de la educación universitaria a un número mucho mayor de estudiantes, al menor costo posible, y para trabajar conjuntamente por la responsabilidad académica y la autonomía intelectual de estudiantes y profesores, todo esto mediante el desarrollo y el uso de las tecnologías de la información y las telecomunicaciones.

La red o sistema fue fundado hace aproximadamente dos (2) años en Dallas, Texas, Estados Unidos, por el señor Randy Best, destacado empresario y filántropo de la educación, quien para conformarla reunió a un destacado grupo de educadores y empresarios.

Entre estos profesionales cabe destacar a los siguientes:

- El Dr. Gerald A Heeger, politólogo de la Universidad de California-Berkeley; máster y $\mathrm{PhD}$ de la Universidad de Chicago; ex rector de la Universidad de Maryland, quien es actualmente el Presidente de Whitney.

- El Dr. Rod Paige, educador graduado de la Universidad de Jackson State y $\mathrm{PhD}$ de la Univesidad de Indiana; ministro de educación de los Estados Unidos entre los años 2000 y 2004, quien es el más alto consejero de Whitney.

- El Dr. Vernon Johnson, educador y administrador educativo con títulos de la Universidad de Ball State y $\mathrm{PhD}$ de la Universidad de Indiana, quien es el Vicepresidente Ejecutivo de Desarrollo Educativo de Whitney. 
- El Sr. Darab Ganji, máster de la Universidad de Texas, quien es el Vicepresidente Ejecutivo de Desarrollo Estratégico de Whitney.

- El Sr. Greg Campbell, profesional de la Universidad de Cornell y M.B.A de la Universidad de Harvard, quien es el Vicepresidente Ejecutivo del Modelo Educativo Whitney.

- El Sr.Vince Conti, profesional y máster de la Universidad de Pensilvania, quien es el Oficial Jefe de Operaciones de Whitney.

- El Sr.Tom Evans, contador certificado en los Estados de Ohio y Texas, quien es el Oficial Jefe Financiero de Whitney.

- El Dr. G. Reid Lyon, PhD de la Universidad de Nuevo México, quien es el Vicepresidente Senior de Investigación y Evaluación de Whitney.

- El Sr.Jorge Ferrari, profesional cum laude de la Universidad de Estados Unidos de México, y máster de la Universidad de Columbia, quien es el Vicepresidente Senior para América Latina de Whitney.

Y otros diez (10) directivos del más alto nivel, igualmente con amplia trayectoria académica o administrativa.

Una de las premisas fundamentales del sistema Whitney es que las tecnologías de la información y las comunicaciones, que avanzan vertiginosamente, serán factor esencial del aumento de la cobertura de la educación superior y del desarrollo progresivo de la autonomía intelectual de todos los miembros de las comunidades académicas. Por esta razón Whitney viene trabajando en varios proyectos tecnológicos, de los cuales quisiera mencionar dos en esta ocasión.
En primer lugar, el desarrollo de un nuevo modelo educativo que combina metodologías educativas presenciales, menos presenciales, semipresenciales, a distancia y virtuales, el cual resulta especialmente propicio para el desarrollo de la autonomía intelectual, pues el estudiante tiene que demostrar autónomamente el cumplimiento de sus metas académicas, con la ayuda de sus profesores. Poco a poco se incrementarán el uso de videoconferencias y la ayuda de profesores tutores permanentemente disponibles a través del computador.

En segundo lugar, el desarrollo y construcción de un computador de bajo costo para fines académicos. Recientemente nos entregaron ya un primer prototipo que tiene un costo de 418 dólares y creen que llegarán a producirlo en alrededor de 300 dólares. Con este proyecto se pretende que en un futuro próximo todos los estudiantes tengan su propio computador, conectado vía satélite, por medio del cual podrán demostrar el cumplimiento de sus logros académicos y mantenerse en contacto con sus profesores y tutores.

La red Whitney ofrecerá valiosas oportunidades a sus instituciones vinculadas, tales como las siguientes:

1) Intercambios de profesores y estudiantes. De hecho, la movilidad estudiantil de estudiantes del Politécnico Grancolombiano hacía otros países venía incrementándose sustancialmente en los últimos años.

2) Compartir experiencias académicas y administrativas. A manera de ejemplo, el Politécnico desde su fundación viene desarrollando su propio sistema de información académica, que llamamos SIA, de acuerdo con sus iniciales, $\mathrm{y}$ ahora tendremos mejores opciones de 
perfeccionarlo y ofrecerlo a otras instituciones.

3) Realizar economías de escala o ahorros en bienes y servicios que requieren todas las instituciones, como software, seguros, auditorías, etc.

4) Compartir tecnologías educativas, como las que mencioné anteriormente que viene desarrollando el sistema Whitney.

5) Compartir y crear programas académicos técnicos, tecnológicos, profesionales y de posgrado, y también de carácter no formal, dentro de los conceptos de educación superior por ciclos y de educación continuada, que han sido característicos del Politécnico.

6) Colaboración para los procesos de autoevaluación y acreditación tanto nacionales como internacionales.

7) Creación o participación en sistemas de certificación de competencias para demostrar las habilidades laborales que confieren los distintos programas académicos.

8) Crear nuevas oportunidades de educación bilingüe o multilingüe.

En fin, Whitney ofrecerá a sus universidades afiliadas todo un conjunto de alternativas para adecuarse a los requerimientos del mundo en vertiginoso proceso de Globalización.

La Fundación Politécnico Grancolombiano, Institución Universitaria, suscribió su afiliación a Whitney Internacional University System el pasado 16 de agosto de 2006, después de un dispendioso proceso de "due diligence" o diligencia debida que se prolongó por más de un año, en el cual tuvo que demostrar toda su realidad a través de la firma que ejerce su revisoría fiscal, la multinacional Ernst \& Young, que coincidencialmente realiza también la auditoría de la red en los Estados Unidos. Para permanecer en la red, la Fundación tendrá que cumplir las más estrictas y exigentes normas internacionales de gobierno corporativo.

La red se inició con dos universidades americanas, el New England College of Finance, de Boston, y el American College of Education, de Illinois; ya cuenta con instituciones vinculadas en Brasil, México, Argentina, Colombia y Panamá, y actualmente está estudiando postulaciones de instituciones de otros países, como Marruecos, Arabia Saudita y la India. Para el año 2011 Whitney proyecta tener alrededor de 700.000 estudiantes de todo el mundo vinculados a la red.

Es importante reiterar que la pertenencia a este sistema internacional no implica ningún condicionamiento religioso o ideológico. La Fundación Politécnico Grancolombiano seguirá siendo una institución sin ánimo de lucro, de carácter no confesional, pero especialmente respetuosa de todas las religiones e ideologías. Su principio o valor esencial será el mismo de siempre: el espíritu de bondad y de amor al prójimo, que, para que no se le dé solamente una connotación religiosa, prefiero denominarlo sentido de solidaridad, equidad y generosidad. Yo creo que este es el único principio objetivo que es satisfactorio y cabe en todas las religiones e ideologías.

\section{PABLO MICHELSEN NIÑO}

Rector 